\title{
EDITORIAL
}

\section{The realities of the Liverpool care pathway}

W hen writing an editorial, it is usual practice to draw on extensive professional knowledge, communication with experts, and important information obtained from attending relevant international conferences. Indeed, when commissioning this special Focus Issue on Palliative Care, this professional background was what I drew upon. However, the field of palliative care is all too often a space where the personal and professional worlds collide.

The development of this Focus Issue was based on my attendance of the $21^{\text {st }}$ Asia Pacific Cancer Conference in which an entire stream of presentations was devoted to the field of palliative care. This emphasis on the importance of palliative care within the main body of oncology care is not something that I have seen at European and North American conferences, and we deemed it important as a journal to shine a light on this field within the broader oncology community. Although there have been few clinical trials in the field of palliative care, the vast majority of patients who attend an oncology clinic will be in need of palliative care consultation at some point in their treatment trajectory.

As outlined by the articles within this Focus Issue, palliative care treatment has progressed substantially in recent years-many patients receive fantastic care throughout their disease, and afterwards in the form of survivorship care. However, the majority of oncologists worldwide do not have specific training in palliative care or in the communication of the complex issues that are associated with discussions of this kind.

As I alluded to previously, while working on this Focus Issue I was involved in palliative care conversations regarding the treatment of a member of my family. These discussions took place at a hospital in the UK that has been recognized for the quality of its oncology and palliative care; and the quality, care and consideration of the staff were all of a very high level. Based on this experience, I would like to discuss the Liverpool care pathway from the perspective of someone who has learnt about it academically as well as someone who has been confronted with the realities of it.

The Liverpool care pathway was designed in the 1990s to guide the treatment of patients in the last 72 hours of life. One of its central tenants is that of communication with the patient, family and/or primary carers. The pathway was constructed because it was recognized at the time that there were centres of excellence in end-of-life care, but that not all people treating patients at this critical stage had the training to provide optimal care. The aim of the pathway is to enable patients to spend their

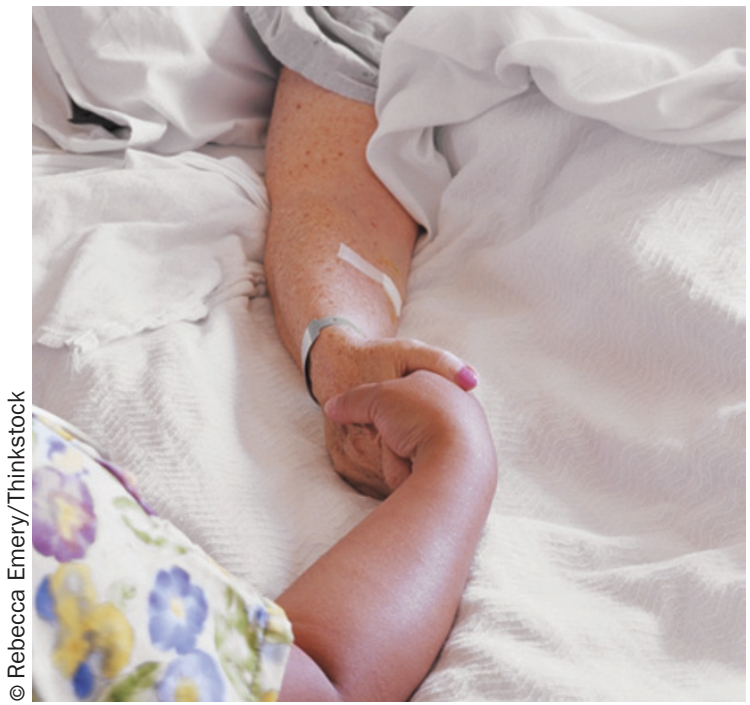

4 For this

to work, the professionals

we are speaking to need to be trained in ... providing palliative care 77 last days as dignified and pain-free as possible. To that end, the pathway has gone through various updates by the Marie Curie Palliative Care Institute since it was first drawn up, and emphasizes training: "a robust continuous learning and teaching programme must underpin the implementation and dissemination of the Liverpool care pathway generic document."

The pathway requires a thorough clinical assessment by a trained multidisciplinary team to determine whether a patient is in the last days or hours of life. Once this conclusion has been reached, the pathway requires a clear communication of the finding to the patient (if possible) and to the family and/or carers. ${ }^{1}$ To assist this discussion, leaflets and documentation have been produced that are intended to convey this important information in as clear and concise a way as possible. However, a report that was picked up by the UK press in late $2012,{ }^{2,3}$ has highlighted that half of patients on the pathway were not aware that they had been placed on it, indicating that communication is a primary issue.

In my case, the clinicians discussing the Liverpool care pathway with the patient and us family members had encountered some people with at least a common language with them (that of medical science). I have never conducted a patient assessment using the Liverpool care pathway, but I know what it is, what its background is, and what it is intended to do. Furthermore, despite reading the newspapers, I had no belief that this pathway was anything like a "death pathway" as described in some areas of the press. ${ }^{3}$ However, the decisions that were required that immediately followed the announcement
Rebecca Kirk is the Senior Editor of Nature Reviews Clinical Oncology.

Competing interests The author declares no competing interests. 
that my family member was on the pathway came thick and fast and were hard to absorb.

Even as trained professionals, being put in a position of deciding how to treat a family member is difficult. For example, the decision of whether or not it is appropriate to sedate a loved one-even only very mildly and even when intended only to reduce distress - is not easy. The communication side of the pathway requires, and expects, that family and carers should be consulted about decisions of this kind. Indeed, it is right and proper that they (we) should be consulted. But, in making these decisions, we need the recommendations and qualified experience of the professionals treating the patient. We need the medical staff to provide options and the anticipated outcomes. Most importantly, we need to have the information explained to us in a language and way that we can take in and understand, and we need to be given the support and time to make these decisions. For this to work, the professionals we are speaking to need to be trained in communication of this kind; they need to be trained in providing palliative care.

The articles in this Focus Issue will add to the body of literature on palliative care targeted to the oncology clinician. They each address a different aspect of that treatment trajectory and are all written by experts. I hope that they will inspire one or two medical students to consider taking a rotation in palliative care, and further inspire those already practicing to learn more about this important aspect of treating their patients as well as their families and carers.

doi:10.1038/nrclinonc.2013.3
1. Marie Curie Palliative Care Institute Liverpool. The Liverpool Care Pathway for the dying patient (LCP) Core Documentation [online], http://www.liv.ac.uk/media/livacuk/ $\mathrm{mcpcil/migrated-files/liverpool-care-}$ pathway/updatedlcppdfs/LCP_V12_Core
Documentation_FINAL_\%28Example\%29.pdf (2009).

2. The Telegraph. Half of those on Liverpool Care Pathway never told [online], http://www.telegraph.co.uk/health/ healthnews/9716418/Half-of-those-
on-Liverpool-Care-Pathway-never-told.html (2012).

3. The Guardian. When should doctors embark upon the death pathway? [online], http:// www.guardian.co.uk/lifeandstyle/shortcuts/ 2012/oct/23/doctors-death-pathway (2012). 